\title{
Structural studies of nanocrystalline thin Pd films electrochemically doped with hydrogen
}

\author{
J. Č́̌žek ${ }^{1 a}$, M. Vlček ${ }^{1}$, F. Lukáč ${ }^{1}$, M. Vlach ${ }^{1}$, I. Procházka ${ }^{1}$, G. Brauer², \\ W. Anwand ${ }^{2}$, A. Mücklich ${ }^{3}$, S. Wagner ${ }^{4}$, H. Uchida ${ }^{4}$, A. Pundt ${ }^{4}$ \\ ${ }^{1}$ Faculty of Mathematics and Physics, Charles University in Prague, \\ V Holešovičkách 2, CZ-18000 Praha 8, Czech Republic \\ ${ }^{2}$ Institut für Strahlenphysik, Helmholtz-Zentrum Dresden-Rossendorf, \\ PO Box 510 119, D-01314 Dresden, Germany \\ ${ }^{3}$ Institut für lonenstrahlphysik und Materialforschung, Helmholtz-Zentrum Dresden-Rossendorf, \\ PO Box 510 119, D-01314 Dresden, Germany \\ ${ }^{4}$ Institut fur Materialphysik, Universität Göttingen, \\ Friedrich-Hund-Platz 1, D-37077 Göttingen, Germany \\ ajakub.cizek@mff.cuni.cz (corresponding author)
}

Keywords: palladium, hydrogen, thin films, positron annihilation.

\begin{abstract}
Hydrogen absorption in Pd causes a significant volume expansion. In free-standing bulk $\mathrm{Pd}$, the hydrogen-induced volume expansion is isotropic. However, the situation becomes more complicated in thin Pd films. Contrary to bulk samples, thin films are clamped to an elastically stiff substrate, which prevents in-plane expansion. Hence, the volume expansion of a thin film is strongly anisotropic because it expands in the out-of-plane direction only. Internal stresses introduced by absorbed hydrogen may become so high that detachment of a film from the substrate is energetically favorable and buckles of various morphologies are formed. In the present work, we studied hydrogen-induced buckling in a nanocrystalline thin Pd film deposited on a sapphire substrate. Slow positron implantation spectroscopy (SPIS) was employed as a principal tool for the characterization of defects and investigation of defect interactions with hydrogen. SPIS studies were combined with X-ray diffraction and direct observations of buckling by light microscopy. It was found that buckling of thin Pd film occurs at hydrogen concentrations $x_{H}>0.1$ and is accompanied by a strong increase of dislocation density.
\end{abstract}

\section{Introduction}

Hydrogen dissolved in interstitial sites in a host metal lattice causes a volume expansion, which is isotropic in free standing bulk samples. However, in thin films, in-plane expansion is hindered by clamping of the film to the substrate. This makes hydrogen-induced expansion strongly anisotropic: the in-plane expansion is suppressed, while the out-of-plane expansion is remarkably larger than in a free standing bulk metal. As a consequence, high compressive bi-axial in-plane stresses up to several GPa occur in thin films loaded with hydrogen [1]. These hydrogen-induced stresses grow with increasing hydrogen content and may cause local or global detachment of the loaded film from the surface, which results in formation of buckles with various morphologies [2-4]. Understanding of hydrogen-induced buckling is highly important since this process may cause catastrophic adhesion failure in many thin film systems or coatings exposed to hydrogen.

Hydrogen-induced buckling occurs when the stored elastic strain energy overcomes the adhesion energy to the surface. Since the formation of buckles represents an irreversible change of film shape (i.e. plastic deformation) one can expect that new defects are introduced during buckling process. Although the morphology of buckles was investigated in a number of works [2-4], there is still a lack of information about defect evolution in buckled films. In the present work slow positron implantation spectroscopy (SPIS) was employed for characterization of development of defects in thin Pd films loaded with hydrogen. Defect studies by SPIS were combined with X-ray diffraction (XRD) and direct observations of buckles by light microscopy. 


\section{Experimental Details}

Thin Pd films were deposited on optically polished (11-20) sapphire substrates by cold cathode beam sputtering in a UHV chamber $\left(10^{-10} \mathrm{mbar}\right)$. The samples were then step-by-step doped with hydrogen by electrochemical charging [5] in a galvanic cell filled with a $1 \mathrm{M} \mathrm{KOH}$ electrolyte. The hydrogen concentration in the loaded sample was calculated from the transported charge using the Faraday's Law and is expressed as the atomic ratio H/Pd throughout this paper. A bulk Pd sample (99.95\%) supplied by MaTecK $\mathrm{GmbH}$ and annealed at $1000^{\circ} \mathrm{C}$ for $1 \mathrm{~h}$ was used as a reference sample.

SPIS studies were performed on the magnetically guided slow positron beam "SPONSOR" [6] with positron energy adjustable from 0.03 to $36 \mathrm{keV}$. Doppler broadening of the annihilation line was evaluated using the $S$ and $W$ line shape parameters, which were always normalized to the bulk values $S_{0}$ and $W_{0}$ determined in the reference Pd sample. Fitting of the dependence of the line shape parameters on positron energy was performed by the VEPFIT software package [7], which solves the positron diffusion-annihilation equation in a single-layer Pd system.

XRD studies of hydrogen-loaded Pd films were carried out using synchrotron radiation with wavelength of $0.499 \AA$ in HASYLAB (DESY) at the beam line B2 [8] equipped with Eurelian cradle Huber 513. The XRD measurements were performed in the Bragg-Brentano symmetrical geometry and the XRD profiles were fitted by the Pearson VII function.

The Transmission Electron Microscopy (TEM) studies were performed on a Philips CM300SuperTWIN microscope operating at $300 \mathrm{kV}$. Thin foils for cross sectional TEM were produced by conventional preparation using a Gatan precision ion polishing system.

A metallographic microscope Arsenal AM-2T was used for observation of buckling in hydrogen loaded films.

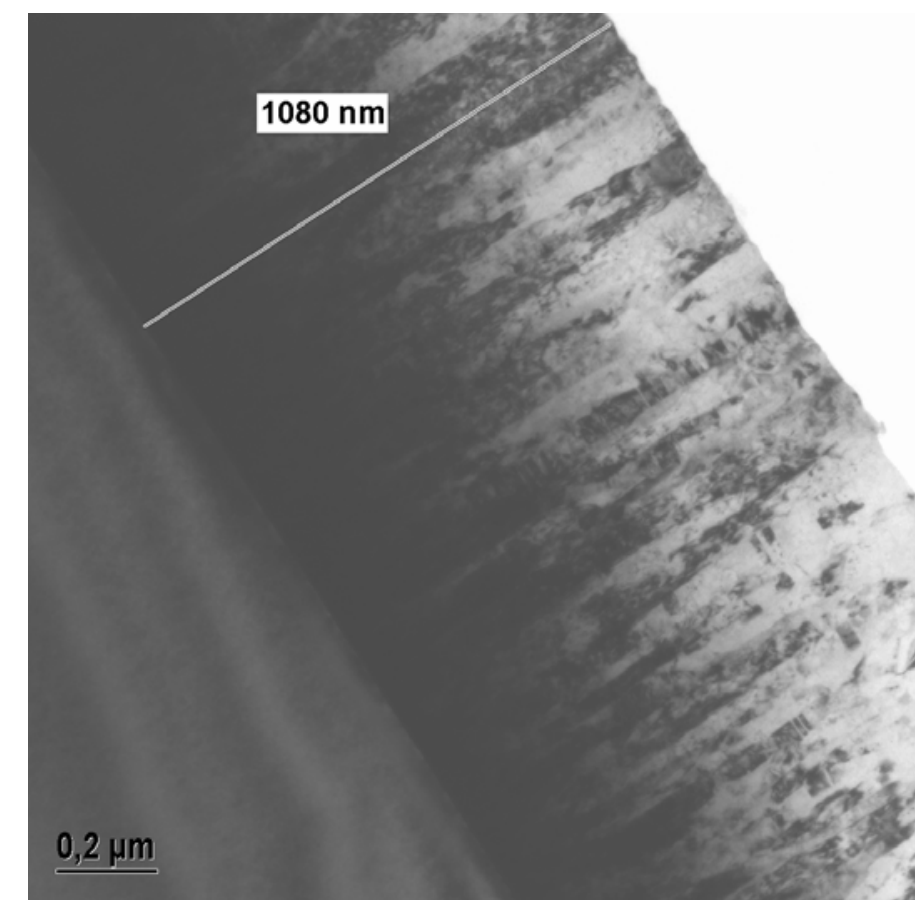

Figure 1 Bright-field TEM image of the virgin Pd film (cross-section).

\section{Results and Discussion}

Virgin film Fig. 1 shows a TEM micrograph in cross section of a virgin Pd film. The film thickness determined by TEM is $1080 \mathrm{~nm}$. The Pd film exhibits nanocrystalline column-like grains having width of $\sim 50 \mathrm{~nm}$. One can distinguish two kinds of columns: (i) 'first generation columns' growing directly on the sapphire surface and (ii) 'second generation columns' growing on the top of the first generation. Fig. 2 shows a TEM image of the first generation columns and corresponding 
electron diffraction pattern. The isolated spots in Fig. 2B with six-fold symmetry arise from the sapphire substrate while the continuous circles represent diffraction from the first generation columns and testify that the Pd film exhibits a fiber (111) texture in the region close to the substrate. A TEM image and electron diffraction pattern for the second generation columns are shown in Figs. $3 \mathrm{~A}$ and $3 \mathrm{~B}$, respectively. The second generation columns exhibit also a fiber (111) texture but it is not as sharp as in the first generation. One can se in Fig. 3B that in addition to (111) fiber texture, additional texture components (e.g. 211) appeared in the electron diffraction pattern.
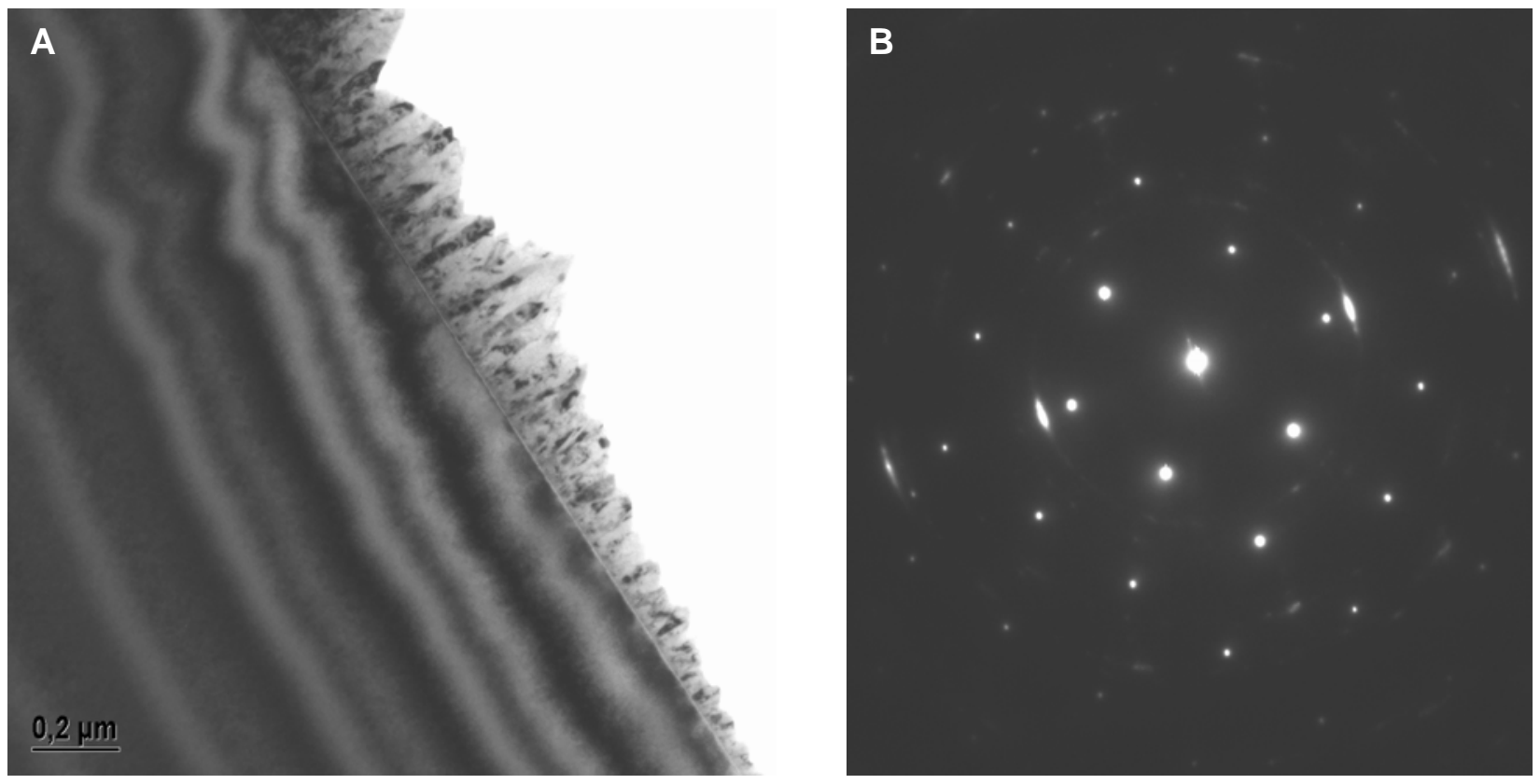

Figure 2 Virgin Pd film: (A) bright-field TEM image, (B) electron diffraction pattern from the first generation columns, i.e. the region close to the substrate.
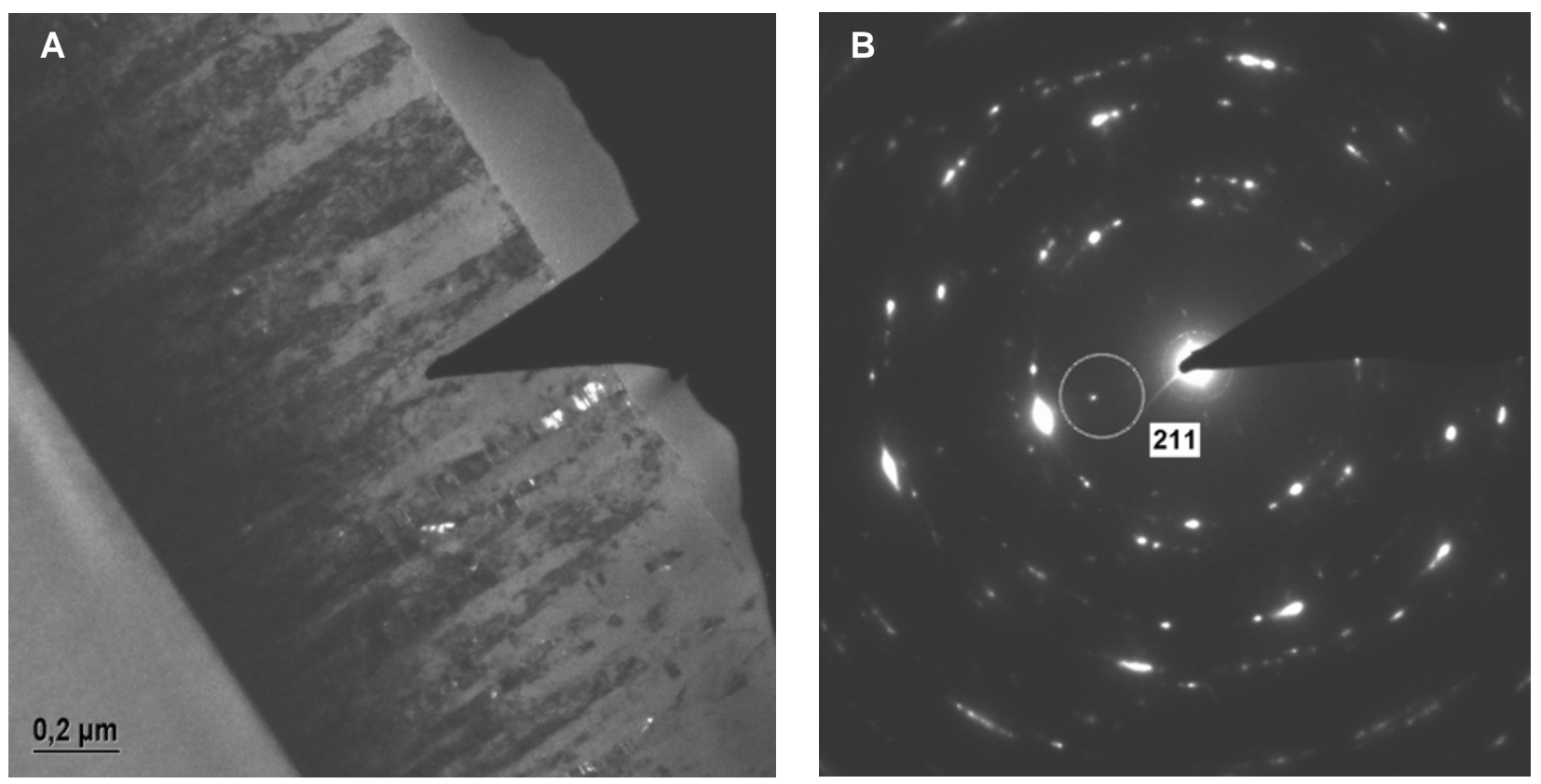

Figure 3 Virgin Pd film: (A) dark-field TEM image, (B) electron diffraction pattern from the second generation columns, i.e. the region close to the film surface. 
The XRD spectrum for the virgin Pd film exhibits a sharp (11-20) reflection from the sapphire substrate and (111) and (222) reflections from the Pd film. This testifies to a fiber (111) texture in the virgin Pd film. Fig. 4 shows the XRD profile of the (111) reflection in the virgin Pd film; the vertical dashed line in the figure indicates position of the (111) reflection in a bulk Pd crystal. As expected, in the Pd film the (111) reflection is shifted to the lower diffraction angles with respect to the position in bulk Pd crystal. This testifies to compressive in-plane stress in the virgin film caused by lattice mismatch between the Pd layer and the sapphire substrate. The Pd film is squeezed in the plane of the substrate in order to match better the inter-atomic distance in the sapphire substrate and to reduce the interface energy between the film and the substrate. Squeezing of the film in the plane of the substrate by a compressive bi-axial in-plane stress simultaneously causes its expansion in the perpendicular out-of-plane direction. Hence, the lattice parameter in the out-of-plane direction, which is measured in the symmetrical Bragg-Brentano geometry, is longer than in a bulk Pd crystal. The highest compressive stress occurs close to the interface with the sapphire substrate, i.e. in the first generation columns, where the film is squeezed most of all. With increasing distance from the substrate the compressive stress decreases and squeezing of the film is more and more relaxed and the lattice parameter approaches the value for a free-standing bulk Pd crystal. One can see in Fig. 4 that the (111) reflection has asymmetrical shape because it is a superposition of the contribution from the strained first generation of columns (short dashed green line) which exhibit a larger out-ofplane lattice parameter and a more relaxed second generation of columns (long dashed brown line) which exhibit a smaller out-of-plane lattice parameter.

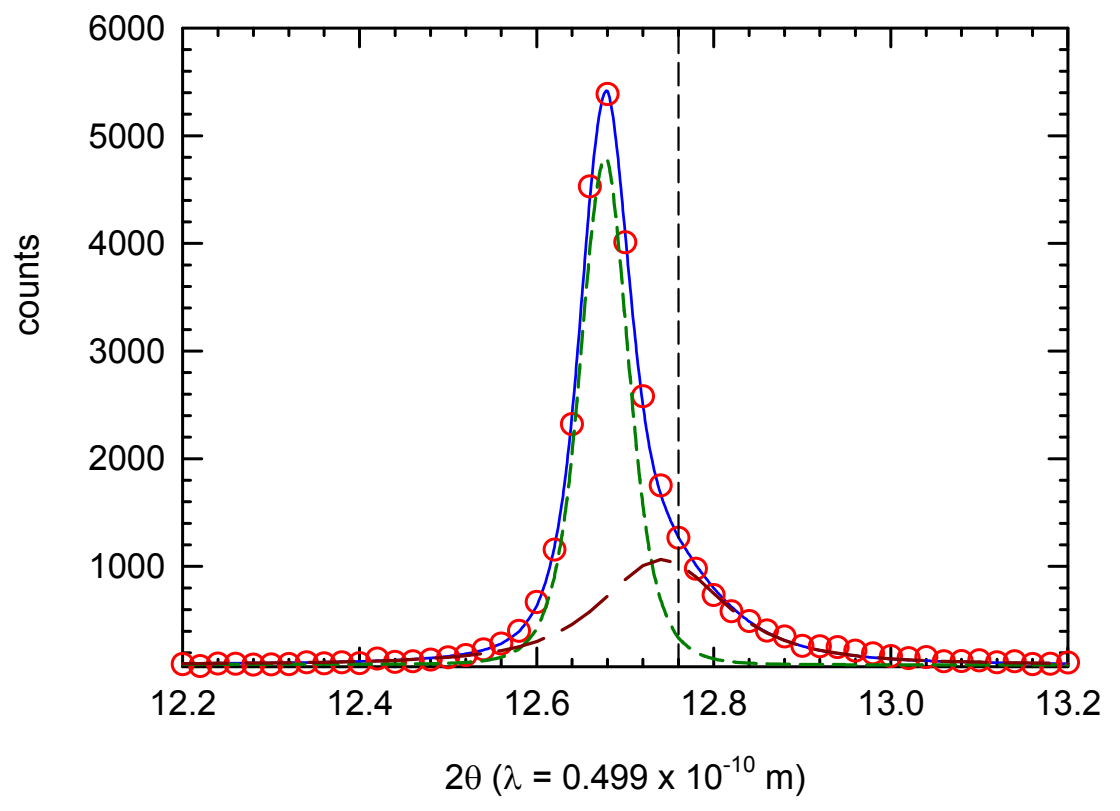

Figure 4 XRD profile of (111) reflection for the virgin Pd film. The solid blue line shows a fit of the XRD profile consisting of a contribution from the first generation columns (short dashed green line) and the second generation columns (long dashed brown line). The vertical dashed black line shows the position of the (111) reflection for a bulk Pd crystal.

Dependence of the $S$ parameter on the positron energy $E$ for the reference well-annealed bulk Pd sample is plotted in Fig. 5. At very low energies positrons annihilate almost exclusively at the surface, characterized by the surface value of the $S$ parameter. With increasing energy positrons penetrate deeper and deeper into the sample and the fraction of positrons diffusing back to the surface decreases. This is reflected by a decrease of the $S$ parameter from the surface value down to a bulk value $S_{0}$ corresponding to a situation when virtually all positrons annihilate inside the bulk $\mathrm{Pd}$. Fitting the $S(E)$ curve for the reference Pd sample by VEPFIT yields the positron diffusion length $L_{+, B}=(151 \pm 4) \mathrm{nm}$. This value is comparable with positron diffusion lengths measured in 
defect-free metals [9] and can be considered as the mean diffusion length of free positrons in a perfect Pd crystal.

The $S(E)$ curve for the virgin Pd film is also plotted in Fig. 5. Compared to the reference wellannealed bulk $\mathrm{Pd}$ sample, the virgin $\mathrm{Pd}$ film exhibits a substantially higher $S$ parameter. Moreover, the mean positron diffusion length of $(41 \pm 5) \mathrm{nm}$, which was obtained from fitting of the $S(E)$ curve for the virgin Pd film, is significantly shorter than in the well-annealed reference bulk Pd sample. The higher $S$ parameter and shorter positron diffusion length give clear evidence that the virgin Pd film contains a significant density of positron traps. This is not surprising since the average width of nanocrystalline columns in $\mathrm{Pd}$ film $(\sim 50 \mathrm{~nm})$ is smaller than $L_{+, B}$. Hence, there is a high probability for positron trapping in open volume defects at interfaces between columns.

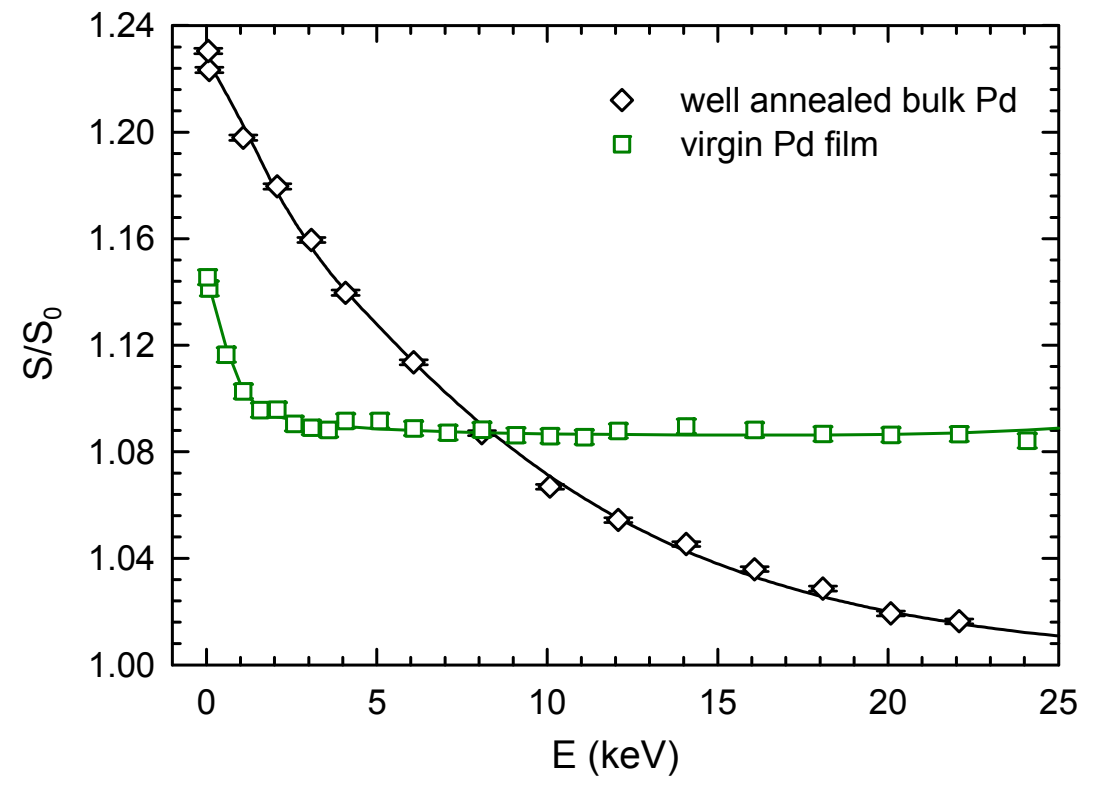

Figure 5 Dependence of the $S$ parameter on the energy of incident positrons for the virgin Pd film and the reference well-annealed bulk Pd sample. Solid lines are model curves calculated by VEPFIT.

Hydrogen-loaded film Light microscopy images of a hydrogen loaded Pd film are shown in Fig. 6. The film surface appears smooth at low hydrogen concentrations $x_{H}<0.10$, see Fig. 6A. The hydrogen concentration $x_{H} \approx 0.10$ seems to be a critical value, above which buckling of the film takes place. Straight buckles are formed in early stages of buckling $\left(0.10<x_{H}<0.15\right)$, see Fig. 6B. Since a straight buckle can release the in-plane stress only in a single in-plane direction the film is forced to expand also in the perpendicular direction [2]. As a consequence in the further stage of buckling $\left(0.15<x_{H}<0.20\right)$ the buckles become curved, see Fig. 6C. In the final stage of buckling $\left(0.20<x_{H}<0.35\right)$ a lot of buckles of undulated shape can be observed in the film, see Fig. 6D. At a hydrogen concentration $x_{H}>0.35$ the film was completely detached from the surface.

Fig. 7 shows selected XRD profiles of (111) reflection for hydrogen-loaded Pd films. The XRD profiles in hydrogen-loaded films remain asymmetric, which testifies that the difference between the first and the second generation columns remain during hydrogen loading. The XRD profiles were, therefore, fitted as a sum of two contributions corresponding to diffraction in the first and second generation columns. The lattice parameters in the first and second generation columns were determined from fitting of (111) and (222) reflection, respectively, and possible misalignment of sample was corrected using the standard Cohen-Wagner plot. Fig. 8 shows the lattice parameters in the first and the second generation columns plotted as a function of hydrogen concentration in the film. 

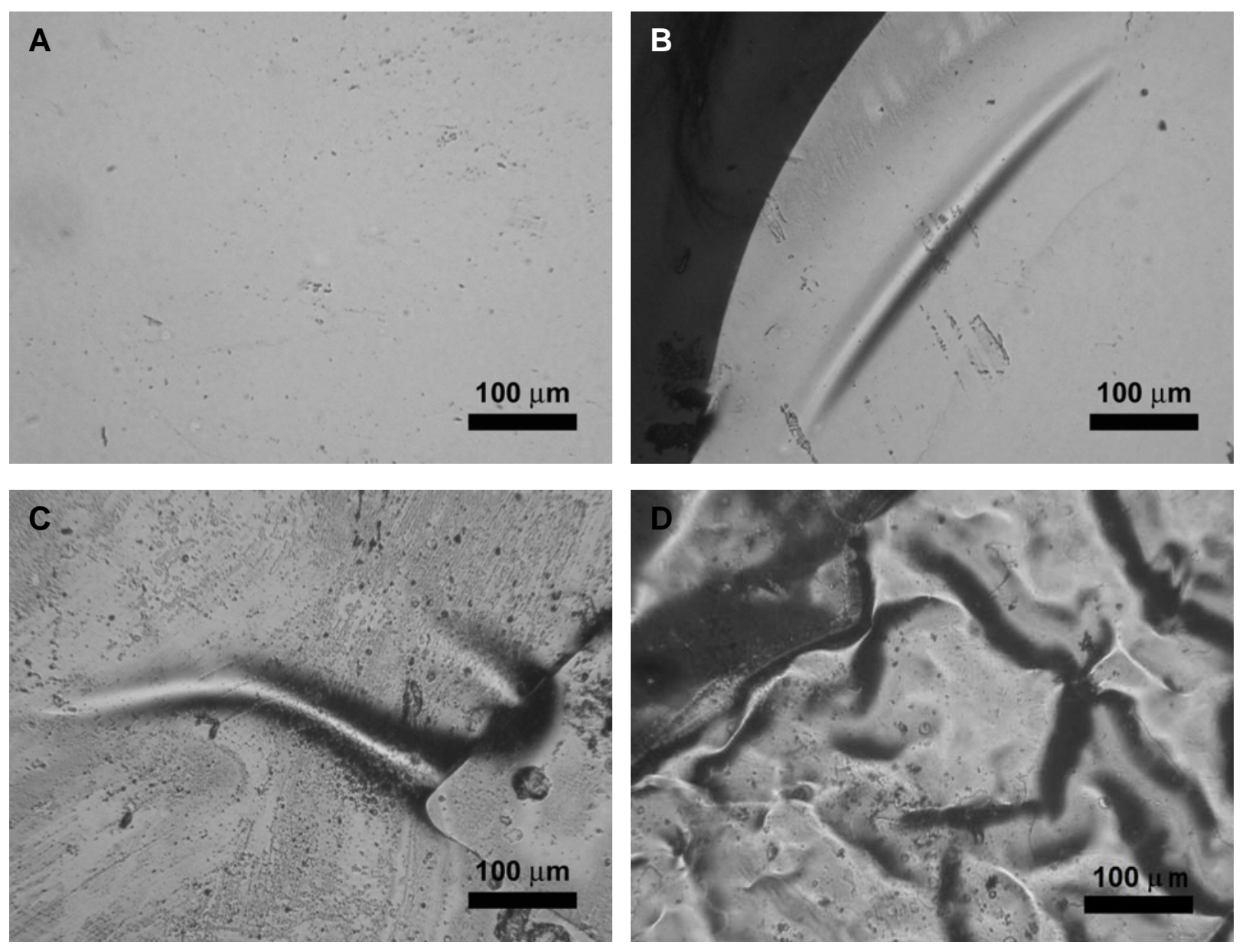

Figure 6 Light microscopy images of the Pd film electrochemically loaded with hydrogen up to various hydrogen concentrations $x_{H}$ : (A) $x_{H}=0.05$, (B) $x_{H}=0.12$, (C) $x_{H}=0.15$, (D) $x_{H}=0.30$.

Absorbed hydrogen causes expansion of Pd film in the out-of-plane direction, which can be seen as a shift of the reflections to lower diffraction angles. An increase of the out-of-plane lattice parameter both in the first and the second generation columns can be clearly seen in Fig. 8. At low hydrogen concentrations $\left(x_{H} \leq 0.02\right)$ the film expansion is fully elastic. However, the hydrogeninduced stresses increase with increasing hydrogen concentration and when the yield stress in Pd is exceeded plastic deformation (i.e. an irreversible change of film shape) takes place. Since hydrogen-induced plastic deformation introduces dislocations into the Pd film the structure of the film becomes less perfect. This is reflected by a broadening and a decrease of intensity of XRD peaks, see Fig. 7. Since hydrogen-induced stress is partially released by plastic deformation, the slope of the increase of lattice parameters was lowered when plastic deformation took place, see Fig. 8. Buckling of the Pd film loaded with hydrogen causes a strong drop in intensity of XRD reflections because of misalignment of the film surface and defocusing. Parts of the films which were detached from the substrate become free and their in-plane expansion is no longer prevented. Hence, detached parts of the film exhibit a strong in-plane relaxation which causes a reduction of the out-of-plane expansion. The effect of the in-plane relaxation due to released compressive stress in the buckled film can be clearly seen in Fig. 8 as a local minimum in the dependence of the lattice parameters on hydrogen concentration. This effect takes place at hydrogen concentration which is close to the onset of hydrogen-induced buckling and occurs earlier and is more pronounced in the first generation columns, which suffer from higher compressive stress imposed by the substrate. After the in-plane relaxation due to hydrogen-induced buckling, the lattice parameter further increases with increasing hydrogen content. The presence of the $\beta$-phase $(\mathrm{PdH})$ with face centered cubic structure and lattice parameter of $(4.017 \pm 0.005) \AA$ was detected at hydrogen concentrations $x_{H} \geq 0.15$. Thus, hydrogen solubility in the $\alpha$-phase in the Pd film is roughly eight times higher than 
in a conventional bulk Pd polycrystal [10]. This effect, which was observed also in other nanocrystalline films [11-14], is due to the nanocrystalline structure of the Pd film, which contains a significant volume fraction of grain interfaces, able to accumulate more hydrogen than grain interiors.

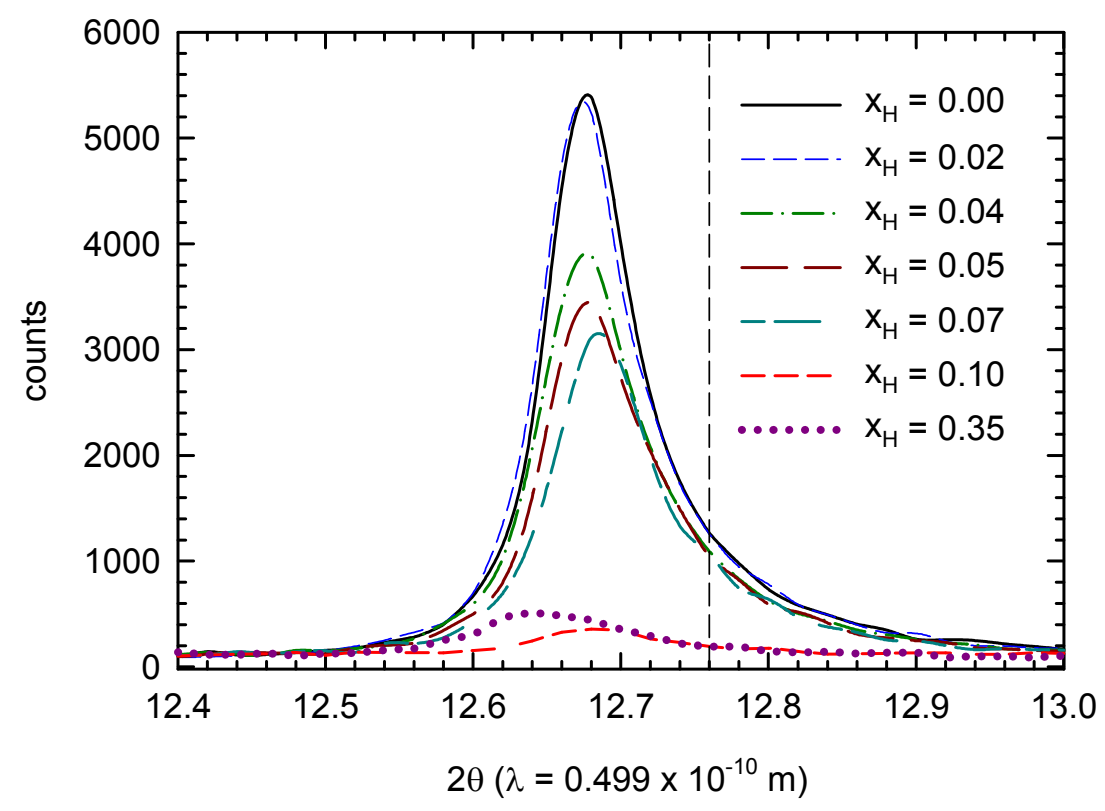

Figure 7 Selected XRD profiles for (111) reflection in Pd films loaded up to various hydrogen concentrations $x_{H}$. The vertical dashed line shows positron of (111) reflection in bulk Pd.

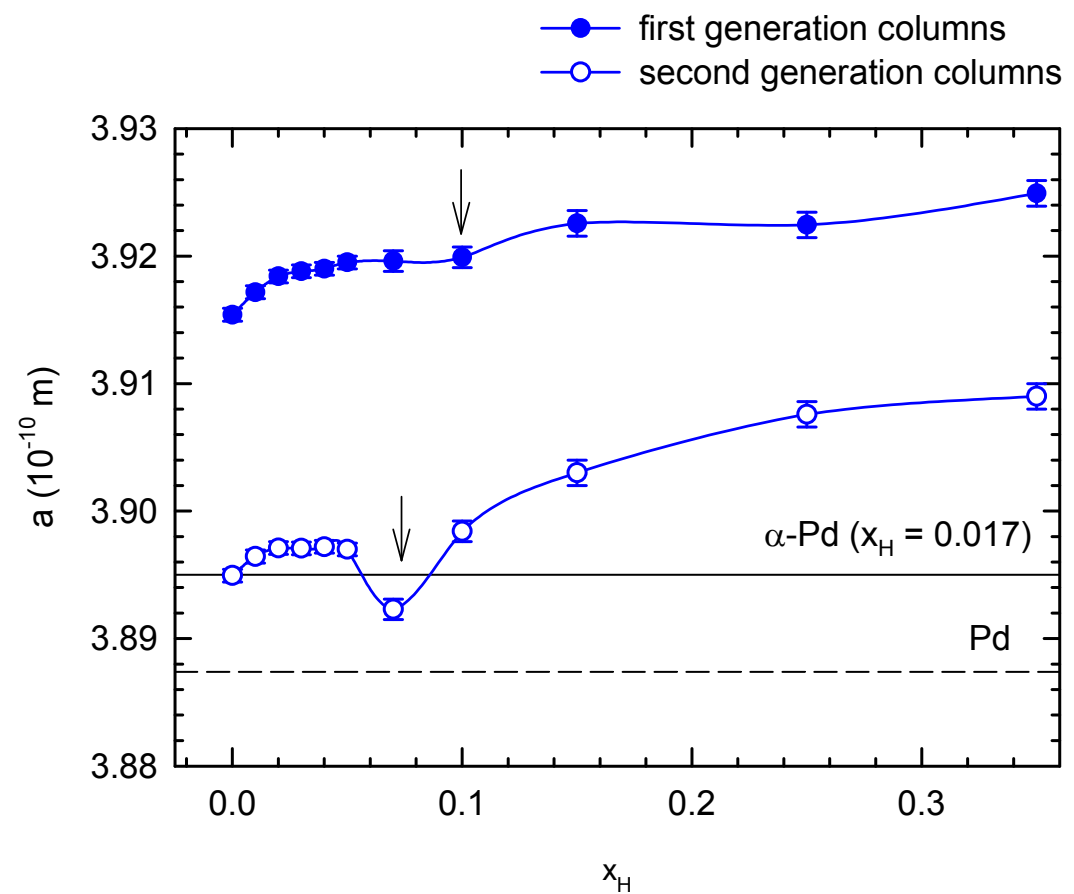

Figure 8 The out-of-plane lattice parameter for the first (full circles) and the second (open circles) generation columns plotted as a function of hydrogen concentration in the film. For comparison, the horizontal dashed and solid line show the lattice parameter for bulk Pd (fcc lattice) and the $\alpha$-phase (fcc Pd with hydrogen concentration $x_{H}=0.017$, which is the maximum hydrogen solubility in the $\alpha$-phase in a conventional bulk Pd polycrystal). The hydrogen concentration when the in-plane relaxation takes place is indicated by arrows. 


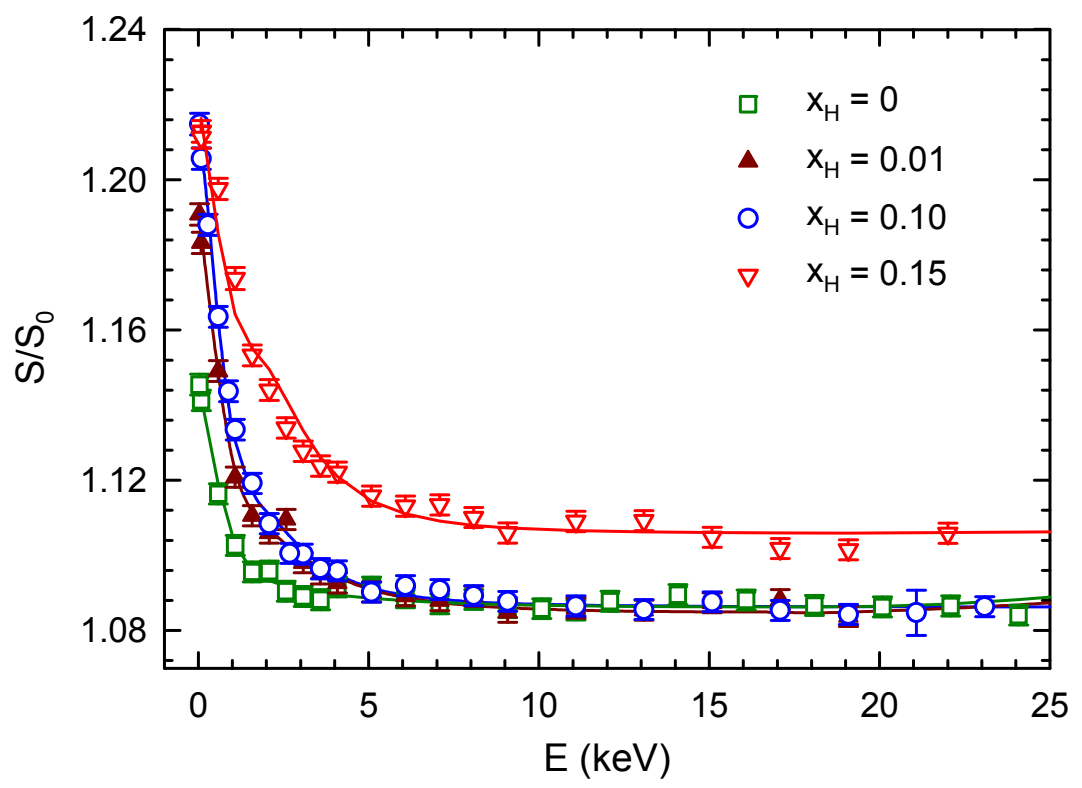

Figure 9 Selected $S(E)$ curves for the virgin Pd film and the film loaded to various hydrogen concentrations $x_{H}$. Model curves calculated by VEPFIT are plotted by solid lines.

Selected $S(E)$ curves for hydrogen-loaded $\mathrm{Pd}$ films containing various concentrations of hydrogen are shown in Fig. 9. The $S(E)$ curves were analyzed by VEPFIT and the calculated model curves are plotted in the figure by solid lines. Fig. 10 shows the $S$ parameter values $S_{P d}$ and the positron diffusion lengths $L_{+, P d}$ for the Pd layer obtained from fitting. The interfaces between nanocrystalline columnar grains contain open volume defects which represent deep trapping sites for absorbed hydrogen atoms. Hence, at low concentrations $\left(x_{H} \approx 0.01\right)$, hydrogen preferentially fills the open volume defects at grain interfaces. Since positrons are annihilated predominantly from trapped state in open volume defects at grain interfaces, SPIS is sensitive to filling of grain interfaces by hydrogen. In Fig. 10 one can observe a decrease of $S_{P d}$ at hydrogen concentration $x_{H} \approx$ 0.01 . This is caused by the filling of deep traps at grain interfaces by hydrogen which leads to reduced localization of the positron wave function due to the repulsive interaction of positron with a hydrogen atom attached to defect. At higher hydrogen concentrations $\left(x_{H}>0.01\right)$, all available deep traps are filled and $S_{P d}$ remains approximately constant. Finally, a dramatic increase of $S_{P d}$ accompanied by a drop of positron diffusion length $L_{+, P d}$ can be seen at $x_{H}>0.10$ when hydrogeninduced buckling takes place. The pronounced increase in $S_{P d}$ and decrease in $L_{+, P d}$ must be due to positron trapping at new defects, presumably dislocations, introduced into the film during buckling. This interpretation is supported by the results in Fig. 11, which shows the $S-W$ plot constructed from the $S$ and the $W$ parameters for Pd films loaded up to various hydrogen concentrations. The bulk $S$ and $W$ parameters for a well-annealed and a cold rolled (plastically deformed) bulk Pd sample are also included. Positron lifetime measurements of the bulk samples revealed that the well-annealed bulk Pd can be considered as a defect-free material, while the cold-rolled bulk Pd contains dislocations. One can see in Fig. 11 that all points fall on a straight line connecting the wellannealed bulk Pd (i.e. defect-free sample) and the buckled Pd film (i.e. the sample with the highest density of defects). This testifies that all samples in the figure contain similar kind of defects (in term of open volume) and differ only by the concentration of these defects. Misfit defects at grain interfaces, which are the dominant trapping sites in the virgin Pd film, exhibit open volume comparable with that of dislocations. Plastic deformation and hydrogen-induced buckling introduce dislocations and this leads to a shift of the corresponding points along the straight line towards higher $S$ and lower $W$ parameters, as indicated by an arrow in Fig. 11. The inset in Fig. 11 shows a detail of the points for hydrogen-loaded Pd films prior to buckling $\left(x_{H}<0.10\right)$. Hydrogen trapped in 
open volume defects at grain interfaces causes a slight reduction of the $S$ parameter and an increase of the $W$ parameter. However, this effect is small compared to hydrogen-induced buckling when a high density of dislocations is introduced into the film and the corresponding point in the $S-W$ plot is shifted to significantly higher $S$ and lower $W$ parameter.

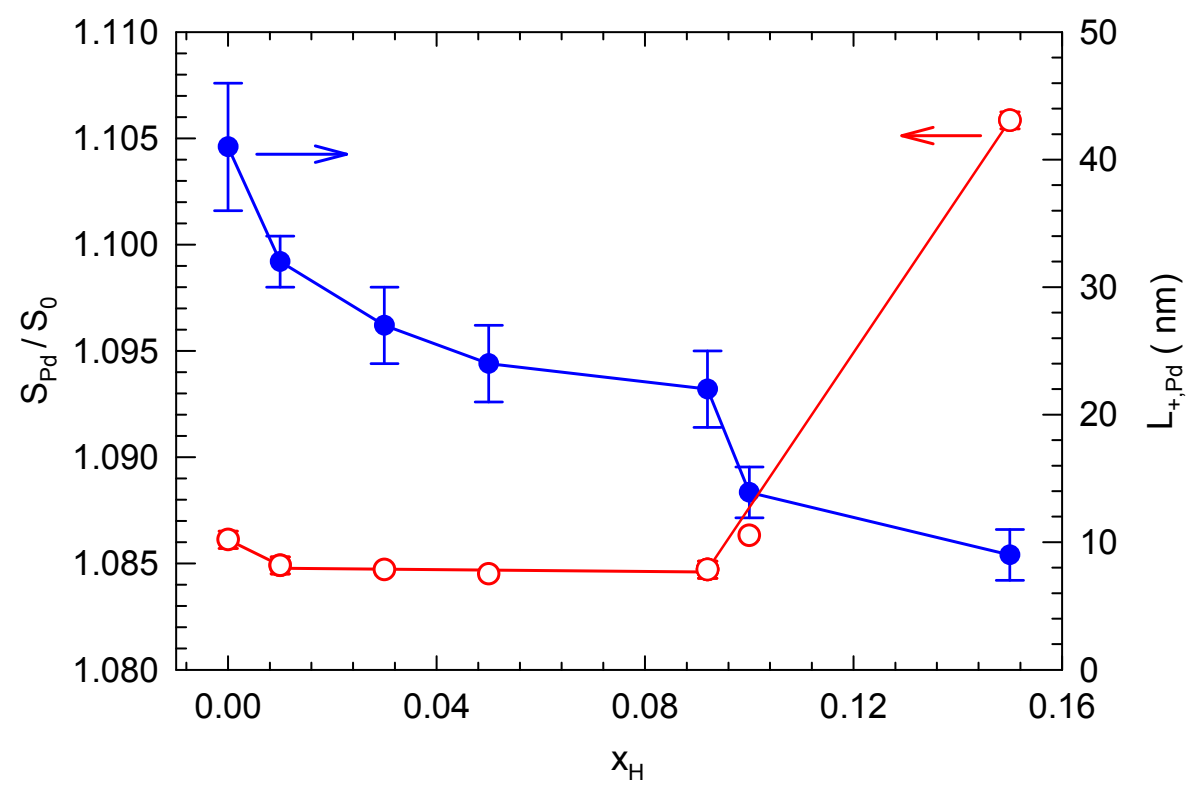

Figure 10 The $S$ parameter $S_{P d}$ (red open circles) and the mean positron diffusion length $L_{+, P d}$ (blue full circles) for the Pd layer plotted as a function of the hydrogen concentration $x_{H}$ in the film.

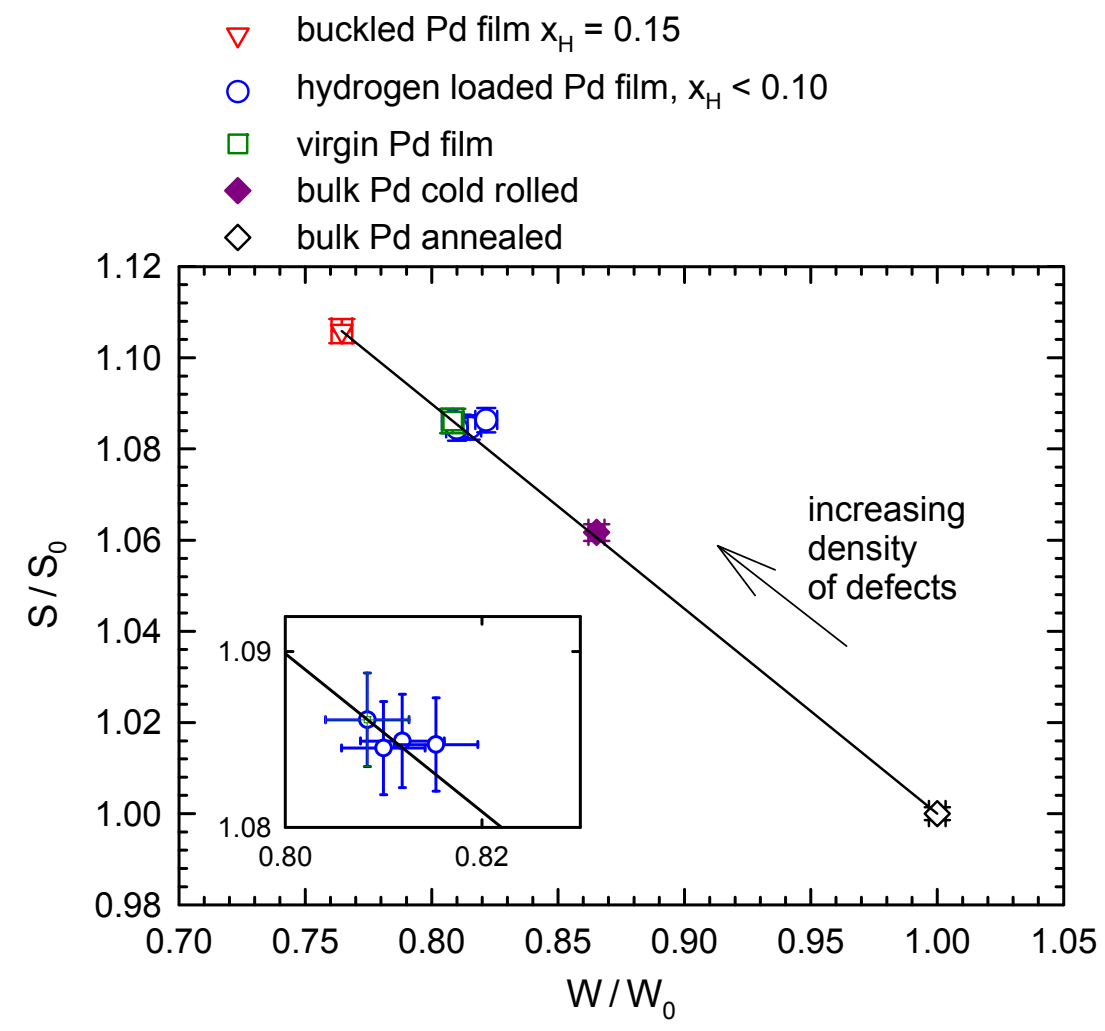

Figure 11 The $S-W$ plot constructed from $S$ and $W$ parameters for Pd films containing various hydrogen concentrations and $S$ and $W$ parameters for a well annealed and cold rolled bulk Pd. The arrow indicates the direction of increasing defect density. The inset in the figure shows a zoomed detail of the points for Pd films loaded with hydrogen prior to buckling, $x_{H}<0.10$. 
Hence, the SPIS results indicate that hydrogen-induced buckling is accompanied by plastic deformation of the film. This is in accordance with acoustic emission (AE) measurements which revealed a huge increase in the number of $\mathrm{AE}$ events when buckling of Pd film occurred [12]. This indicates that collective movement of dislocations takes place during buckling.

\section{Conclusions}

The effect of hydrogen on the microstructure of Pd film deposited on a (11-20) sapphire substrate has been investigated by means of SPIS combined with XRD, TEM and light microscopy. It was found that the virgin film exhibits nanocrystalline columnar grains and bi-axial compressive inplane stress imposed by the sapphire substrate. In the film one can distinguish the first generation columns which are directly attached to the sapphire substrate and suffer from a high compressive stress and more relaxed second generation columns growing on the top of the first generation. Positrons in the virgin film are trapped predominantly at open volume defects at the interfaces between columnar grains.

Hydrogen introduced into the film is firstly trapped at open volume defects at grain boundaries. Subsequently, hydrogen occupies interstitial sites in grain interiors. Absorbed hydrogen causes lattice expansion which is strongly anisotropic because the loaded film expands out-of-plane only, while the in-plane expansion is prevented by clamping of the film to the substrate. This causes a strong increase of the compressive in-plane stress and when hydrogen-induced stress exceeds the film adhesion to the substrate detachment of the film from the substrate becomes energetically favorable and buckling of the film takes place. Detachment of the film from the substrate allows for in-plane relaxation which was detected by XRD. SPIS studies revealed that hydrogen-induced buckling is accompanied by plastic deformation and a high density of dislocations is introduced into the buckled film.

\section{Acknowledgement}

This work was supported by the Ministry of Schools, Youths and Sports of the Czech Republic (project MEB101102), the Czech Science Foundation (project P108/12/G043) and the grant SVV2010-261303.

\section{References}

[1] U. Laudahn, A. Pundt, M. Bicker, U. Hülsen, U. Geyer, T. Wagner, R. Kirchheim, Hydrogeninduced stress in Nb single layers, J. Alloys Comp. 293-295 (1999) 490-494.

[2] A. Pundt, E. Nikitin, P. Pekarski, R. Kirchheim, Adhesion energy between metal films and polymers obtained by studying buckling induced by hydrogen, Acta Mater. 52 (2004) 15791587.

[3] Y. Yu, C. Kim, S.C. Sanday, Buckle formation in vacuum-deposited thin films, Thin Solid Films 196 (1991) 229-233.

[4] A. Pundt, P. Pekarski, Buckling of thin niobium-films on polycarbonate substrates upon hydrogen loading, Scripta Mater. 48 (2003) 419-423.

[5] R. Kircheim, Hydrogen solubility and diffusivity in defective and amorphous metals, Prog. Mater. Sci. 32 (1988) 261-325.

[6] W. Anwand, H.-R. Kissener, G. Brauer, A magnetically guided slow positron beam for defect studies, Acta Phys. Polonica A 88 (1995) 7-11.

[7] A. van Veen, H. Schut, M. Clement, J. de Nijs, A. Kruseman, M. Ijpma, VEPFIT applied to depth profiling problems, Appl. Surf. Sci. 85 (1995) 216-224. 
[8] M. Knapp, C. Baehtz, H. Ehrenberg, H. Fuess, The synchrotron powder diffractometer at beamline B2 at HASYLAB/DESY: status and capabilities, J. Synchrotron. Rad. 11 (2004) 328-334.

[9] P.J. Schultz, K.G. Lynn, Interaction of positron beams with surfaces, thin films, and interfaces, Rev. Mod. Phys. 60 (1988) 701-779.

[10] F. B. Mancher, Phase Diagrams of Binary Hydrogen Alloys, ASM International, Materials Park, 2000, p. 158.

[11] A. Pundt, R. Kirchheim, Hydrogen in metals: microstructural aspects, Annual Review Materials Research 36 (2006) 555-608.

[12] J. Č́žžek, I. Procházka, S. Daniš, O. Melikhova, M. Vlach, N. Žaludová, G. Brauer, W. Anwand, A. Mücklich, R. Gemma, E. Nikitin, R. Kirchheim, A. Pundt, Positron annihilation study of hydrogen trapping at open-volume defects: Comparison of nanocrystalline and epitaxial Nb thin films, J. Alloys Comp. 446-447 (2007) 484-488.

[13] T. Mütschele, R. Kirchheim, Hydrogen as a probe for the average thickness of a grain boundary, Scripta Metall. 21 (1987) 1101-1104.

[14] J. Č́žžek, I. Prochazka, M. Vlach, N. Žaludová, P. Dobroň, F. Chmelík, G. Brauer, W. Anwand, A. Mücklich, E. Nikitin, R. Gemma, A. Pundt, R. Kirchheim, Multi-Scale Analysis of Hydrogen-Induced Buckling in Pd Films, Proc. Eng. 1 (2009) 99-103. 